\title{
Risk and the inadequacy of science
}

There are many aspects to risk, both objective and subjective. Difficulties in quantification, compounded by conflicts in value judgements, necessitate increased openness in its assessment, while avoiding oversimplification.

FOR scientists working on phenomena related to risk, 1996 proved to be an unusually challenging year. Climatologists exchanged heavy bombardment with industrial lobby groups, and advisers on the genetic modification of crops and on spongiform encephalopathies found themselves caught up in issues in which important groups (not to mention nations) had much at stake (see pages 6-11). The way forward in such debates, one might think, is to analyse and quantify the risks at the heart of them, and use the results as a basis for prioritizing problems and drawing up solutions. The plea from a British government minister that scientists develop an equivalent of the Richter scale for risks is a reflection of the need for such communicable insight. But the closer one looks at many risks - especially those giving rise to controversy - the more elusive attempts to summarize them seem to become. The minister is doomed to disappointment in his apparent naiveté, but there are constructive ways forward nevertheless.

The quantification of risk would seem to be straightforward enough when applied to a well-understood technology. For example, as was usefully reviewed in the 1992 report of the United Kingdom's Royal Society, Risk: analysis, perception and management, engineers can model and test pathways by which a given hazard might arise in industrial plant, and biologists can similarly estimate the impacts of toxins and drugs on the human or animal body.

But judgements have to be made at many stages, even in supposedly objective studies. Extrapolation from animal models to human impacts of drugs carries uncertainties; apparently reasonable assumptions can turn out to be disastrously wrong witness, for example, the significant underestimate of the impact of Chernobyl fallout on livestock due to faulty extrapolation of mobilities of elements in clays, or the even more notorious misjudgements underlying the explosion of NASA's Challenger shuttle. As was emphasized by the US National Research Council in its recent report Understanding Risk, even selecting the appropriate measure of impact needs careful consideration: accidental deaths of employees per ton produced by the US coal industry have been falling in recent years, but deaths per employee have been rising. And where mechanisms are poorly understood and epidemiological data sparse, as is the case in the various types of Creutzfeldt-Jakob disease and the associated prion hypothesis, then it is inevitable that scientific judgement is uncomfortably imprecise. In short, even highly objective analysis of risks is overlaid by judgements that need to be scrutinized.

Then comes the problem of perception, and what the insiders call "multidimensionality", leading to different responses by people to a perceived risk. A commonly cited diagram in risk studies places specific hazards in a space whose $x$-axis runs from poorly to well known or understood, and whose $y$-axis ranges from controllable or voluntary to uncontrollable and involuntary. Almost regardless of relative probabilities, risks are much more likely to be accepted if they lie in the bottom left quadrant (familiar and voluntary), for example sports, than if in the top right - radioactive waste and genetic manipulation. And, of course, official quantifications of risk will be distrusted by the public once experts have been proved wrong (Chernobyl fallout,
BSE, infected blood) or if those responsible for quantifying the risks have strong vested interests (the nuclear industry in waste disposal, Shell with the Brent Spar, Ciba-Geigy with genetically modified corn). As the Challenger disaster illustrated, even the best-intentioned and well-resourced organizations can tend to severely underestimate risks in complex new technologies.

Most people who worry about these things have concluded that openness and participation by non-scientists in the process of risk evaluation is essential. It would be misleading to call this a consensus-generating process. A detriment to some can be a benefit to others - another aspect of risk's multidimensionality. Agreement over how to apply value judgements in evaluating such risks is bound to be elusive - witness debates between developed and developing countries on the value of human lives potentially lost through climate change. But those involved in providing scientific advice have found that including non-experts at the outset sets a broader scientific agenda that gives rise to advice that is all the more useful. The use of peer review and the circulation of preliminary drafts of advice can highlight assumptions that are hidden or poorly accounted for. And the involvement of interest groups ensures not only that all viewpoints have had their say but also that a wider appreciation of the factors underlying the risks and their uncertainties has been gained.

As the NRC's report emphasizes, such openness and participation will not solve all the problems - partly because of differences of principle amongst those involved, and partly because it can be impossible to take into account those whose agenda is directly at odds with the project. A company that feels it has nothing to gain from broad risk estimation and even a lot to lose might choose to boycott the exercise altogether and, instead, hijack the regulatory process that follows it. The communication of risk can also be hijacked, as Greenpeace accomplished in the case of the Brent Spar, making the engineering assessments of risk undertaken by Shell effectively redundant as far as the public were concerned.

All of this shows that attempting to reduce risk to a universal scale is a dangerous notion. On the other hand, understanding of the risks underlying a hazard to public health, or to ecosystems, or to nations, is, after all, an essential part of the political process. There is no alternative to the time and expense of developing such understanding, and communicating it effectively in a way, specific to each case, that allows the media to encapsulate its multidimensional facets. Standards of best practice need to be generated as a goal of regulatory policy. The NRC has provided an excellent step forward in that process, particularly in its report's vivid deployment of examples and case studies.

It is not uncommon to hear scientists (and others) say that, if only people understood the science, their perceptions of risk would be much improved. But that sentiment is misconceived. Lay people can understand the science that they wish to more readily than many scientists seem to credit. What is more critical is that interested scientists and non-scientists alike have examined the components, uncertainties and perceptions of any particular risk. That in turn will focus attention on the relevant science. In that sense, improving the public understanding of risk is a particularly appropriate goal for 1997. 